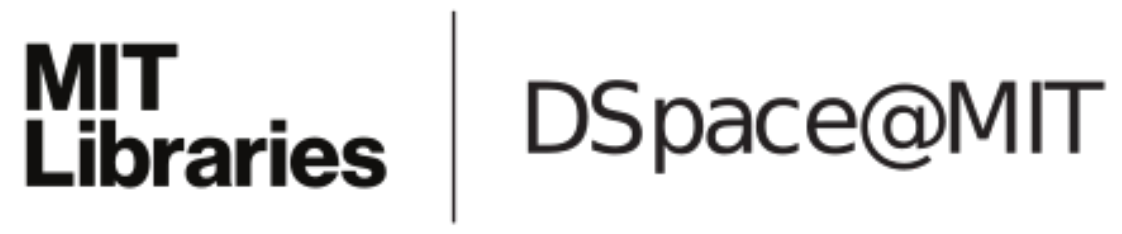

\author{
MIT Open Access Articles
}

Performance Limits of Axial Compressor Stages

The MIT Faculty has made this article openly available. Please share how this access benefits you. Your story matters.

Citation: Hall, D. K., E. M. Greitzer, and C. S. Tan. "Performance Limits of Axial Compressor Stages." Volume 8: Turbomachinery, Parts A, B, and C (June 11, 2012).

As Published: http://dx.doi.org/10.1115/GT2012-69709

Publisher: ASME International

Persistent URL: http://hdl.handle.net/1721.1/116400

Version: Final published version: final published article, as it appeared in a journal, conference proceedings, or other formally published context

Terms of Use: Article is made available in accordance with the publisher's policy and may be subject to US copyright law. Please refer to the publisher's site for terms of use. 


\title{
PERFORMANCE LIMITS OF AXIAL COMPRESSOR STAGES
}

\author{
D. K. Hall, E. M. Greitzer, C. S. Tan \\ Gas Turbine Laboratory \\ Massachusetts Institute of Technology \\ Cambridge, Massachusetts 02139 \\ Email: dkhall@mit.edu
}

\begin{abstract}
This paper presents a framework for estimating the upper limit of compressor stage efficiency. Using a compressor stage model with a representative design velocity distribution with turbulent boundary layers, losses are calculated as the sum of selected local irreversibilities, rather than from correlations based on data from existing machines. By considering only losses that cannot be eliminated and optimizing stage design variables for minimum loss, an upper bound on stage efficiency can be determined as a function of a small number of stage design parameters. The impact of the stage analysis results are evaluated in the context of gas turbine cycle performance. The implication from the results of the stage level and cycle analyses is that compressor efficiency improvements that result in substantial increases in cycle thermal efficiency are still to be realized.
\end{abstract}

\section{NOMENCLATURE}

AR Aspect ratio

$C_{D} \quad$ Dissipation coefficient

$C_{s} \quad$ Camber length

$D$ Diffusion factor

$h$ Enthalpy

$\ell$ Streamwise coordinate

$\dot{m}$ Mass flow rate

$p$ Pressure

$r$ radius

Re Reynolds number

$s$ Entropy

$T$ Temperature

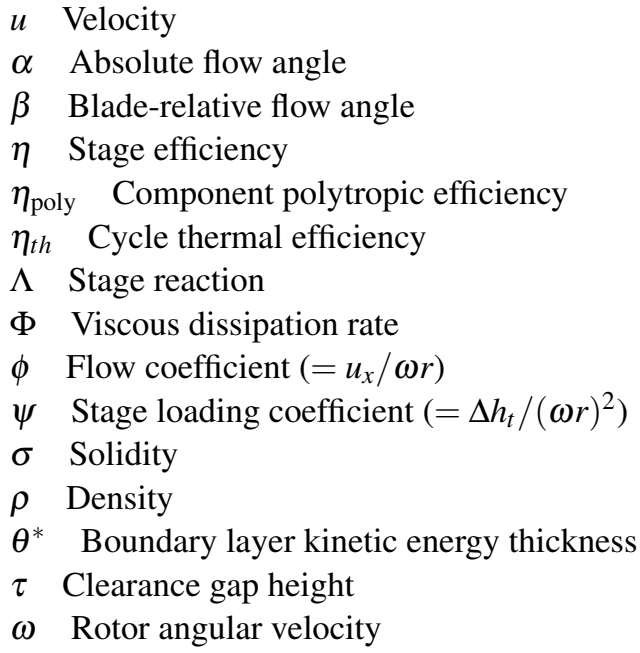

\section{Subscripts}

$e$ Boundary layer edge quantity

$t$ Stagnation quantity

\section{INTRODUCTION}

In this paper, we provide estimates of the limits on axial compressor stage efficiency. The overall context is in the development of a framework to assess the potential for contributions to gas turbine engine performance. The estimates are based on physical principles that govern irreversible processes within a turbomachine, rather than extrapolation of available data. Although analyses of this type may exist in a proprietary context, to 
the authors' knowledge no such estimates exist in the open literature. A central aim in the presentation of the work, therefore, is to provide a forum for discussion of opportunities for increasing efficiency in terms of specific processes.

It is useful at the outset to describe the scope and purpose of the work, because that frames many of the choices about which effects to include and the level of detail needed in the analyses and computations. Previous work on compressor efficiency can be roughly divided into two types of approach. The first is concerned with estimates of the efficiency of real or proposed stages. A notable example of this is given by the several papers from Smith and his co-workers [1] [2] [3]. Another recent example is by Wright and Miller [4]. In these treatments, analyses and correlations are developed for stage performance, with modifications made based on data from test rigs and engines. The resulting methodology gives designers an excellent assessment of the potential performance for state-of-the-art machines, but it does not provide information on the upper bounds of compressor performance.

The second approach focuses on the fundamental irreversible flow phenomena responsible for losses in efficiency. A landmark in this category of analysis is Denton's [5] comprehensive treatment of individual loss mechanisms, which lays out procedures for estimating various losses using first-principles fluid dynamic analysis. Other analyses of this type, by Storer and Cumpsty [6] and Yaras and Sjolander [7], present tip leakage loss models for compressor and turbine stages, respectively, and Young and Wilcock [8] have provided a model for losses due to turbine cooling flows and other flow injection processes. The common thread in the quantification of losses in these treatments is control volume analyses of the phenomena of interest, enabling loss estimates based on models of irreversible processes that do not rely on information about flow details.

The present work fits into this second type of approach. We seek to determine the upper limit of stage efficiency through estimates of the minimum loss arising from irreversible flow phenomena that cannot be eliminated. The paper is organized as follows. We first provide an overview of the ground rules under which this study has been carried out, i.e., definition of the losses considered and assumptions included in the model. It will be seen that there are a number of choices to be made concerning what mechanisms should be included and what should be omitted. We present the underlying views that have led to the choices made because, in a very real sense, this is the crux of the approach and the most difficult part. Second, we present a compressor stage model, consisting of a generic geometry and velocity distributions. This in employed with the goal of developing useful estimates of stage efficiency as a function of a manageable number of stage input parameters. Finally, efficiencies for optimized stages representing a range of aero engine compressor stages are presented, and the overall benefit in terms of gas turbine thermal efficiency is evaluated.

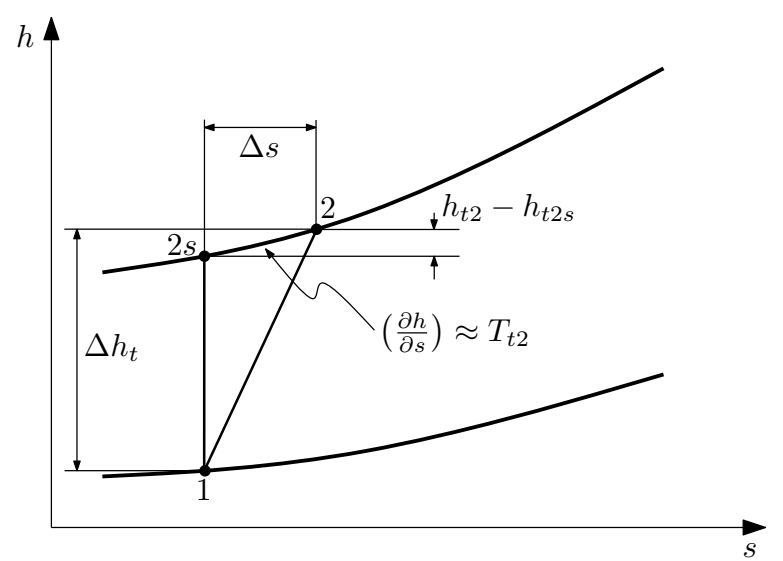

FIGURE 1: IRREVERSIBLE COMPRESSION PROCESS THERMODYNAMICS

\section{APPROACH}

The issue of determining a meaningful upper bound on stage performance is framed by the metrics used to define performance, the losses included in the analysis, and the assumptions made about the flows through the machine.

A key concept is the link between efficiency and entropy generation. For small pressure rises compared to the inlet level, the stage efficiency can be expressed in terms in the entropy generation and the stagnation enthalpy rise (see Figure 1):

$$
\eta=\frac{h_{t 2 s}-h_{t 1}}{h_{t 2}-h_{t 1}} \approx 1-\frac{T_{t 2} \Delta s}{\Delta h_{t}} .
$$

Equation (1) shows that entropy increases through irreversibility, or "lost work," are linked directly to decreased efficiency. We thus focus on the mechanisms for entropy generation.

The following sources of entropy generation represent irreversibilities inherent in flow through compressors; while they can be reduced, they cannot be eliminated:

1. viscous dissipation near solid surfaces (within boundary layers) on blades, vanes, and end walls,

2. mixing of wakes downstream of blade rows,

3. mixing of the leakage flow over the rotor tip gap with the main flow.

These are the only loss sources considered here. The losses that are not included, most notably losses due to shocks and threedimensional effects such as corner separation and stator fixing configuration, are neglected. This is part of the basic choice alluded to previously, and thus deserves some comment. Some of these losses are dependent on the detailed design of the stage; for example, shock losses can be modeled as a function of Mach number, but depending on the design, this loss may be reduced or 
eliminated through selection of blade Mach number. For others the complexity arising from modeling them was deemed unjustified compared to the gain in fidelity they might provide. Where possible, however, estimates of the magnitudes of these "missing" losses are given in the subsequent discussion.

The loss magnitudes for the stages examined are determined from a combination of two-dimensional models. Profile losses over the span of rotor blades and stator vanes are assumed to be captured by a mean-line analysis. Tip leakage and end wall losses are determined from two-dimensional control volume analyses. Three-dimensional flow features (e.g., passage and tip leakage vortices) are not modeled, consistent with the choices made about which losses to include. ${ }^{1}$

The flow is taken as incompressible. Compressibility effects can decrease performance through increased dissipation in boundary layers or through the presence of shocks [9] [10]. Correlations based on test data by Koch and Smith [1], however, indicate that increased boundary layer losses at stage inlet Mach numbers as high as 1.5 result in losses in efficiency of less than one percent; shock losses were also shown to be small relative to profile losses at Mach numbers up to 1.2. Because the magnitudes of these losses are not large, may depend on the detailed design of the stage (e.g., airfoil loading distribution), and are generally most important for the front few stages of a machine, neglecting them is consistent with our approach to estimating maximum efficiency; the incompressible case provides an upper bound on the attainable efficiency while eliminating Mach number as a design parameter.

Boundary layers are taken to be steady and turbulent. In practice, blade boundary layers are unsteady with a portion of laminar flow [9] [11] [12]. Further, the possible unsteady recovery in wake mixing can result in decreased wake loss [13]. We have neglected these effects; the result is an underestimation of the efficiency limit, but this is deemed acceptable in view of the number of parameters needed to describe the unsteady transition process and wake recovery. The analysis can thus be regarded as conservative, but it will be seen that even with these assumptions, there is substantial potential for increases in efficiency.

\section{STAGE DESCRIPTION}

A stage consists of a row of rotating blades (rotor) followed by a row of stationary vanes (stator). The nomenclature is given in Figure 2. For the purposes of determining blade surface area, airfoils are modeled as having negligible thickness and circular arc camber lines. The blade spacing is characterized by the solidity $\sigma=c / s$, where $c$ is the blade chord, and $s$ is the spacing between blades at the mean line radius. The annular geometry is characterized by the aspect ratio $\mathrm{AR}=\left(r_{o}-r_{i}\right) / c$ and hub-to-tip

\footnotetext{
${ }^{1}$ One view of this assumption is that future designs will mitigate, or perhaps eliminate, losses due to three-dimensional effects.
}

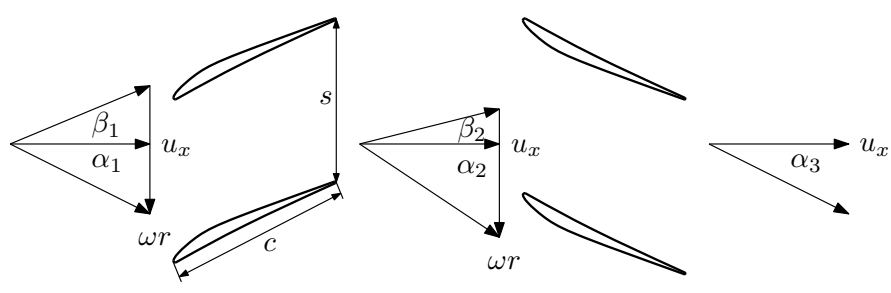

(a) Mean-line profile geometry

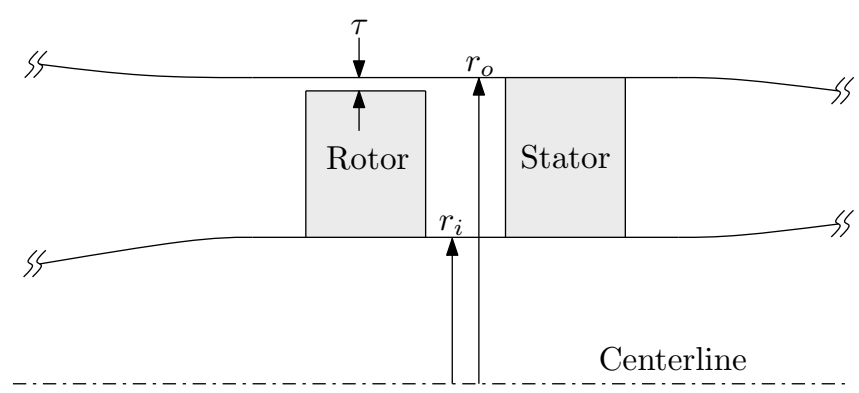

(b) Meridional geometry

FIGURE 2: COMPRESSOR STAGE GEOMTRY

ratio $r_{i} / r_{o}$, both of which are assumed constant. Axial spacing of the blade rows is not considered, since it may be set based on stability or mechanical constraints; the result is an underestimation of the end wall loss approximately equal to the ratio of end wall area in the gap to end wall area in the blade passage. The gap between rotor blade tip and the engine casing is characterized by the gap-to-height ratio $\tau /\left(r_{o}-r_{i}\right)$. No attempt is made to model stator clearance loss, leading to an optimistic result for stage clearance losses.

The compressor is taken to have repeating stages, and as a result, the mean-line velocity triangles are characterized by the flow coefficient $\phi=u_{x} / \omega r$, stage loading coefficient $\psi=\Delta h_{t} /(\omega r)^{2}$, and specification of either the absolute rotor inlet flow angle $\alpha_{1}$ or stage reaction $\Lambda$. The velocity triangles in Figure 2a can be described in terms of these parameters using the Euler turbine equation.

$$
\psi=\phi\left(\tan \alpha_{2}-\tan \alpha_{1}\right)
$$

In determining the dissipation on the blades and vanes, we make use of the velocity distribution used by Dickens and Day [14], with linear changes in velocity from leading edge to trailing edge on both sides of the airfoil. The initial velocity near the leading edge (i.e., following a rapid acceleration after the leading edge stagnation point) is taken as equal to the incoming blade-relative velocity $u_{\text {in }}$ plus or minus some increment $\Delta u$, and the velocity at the trailing edge is equal (on both sides) to the blade-relative velocity just downstream. The inlet and exit velocities are set by the velocity triangles, and $\Delta u$ is determined by 


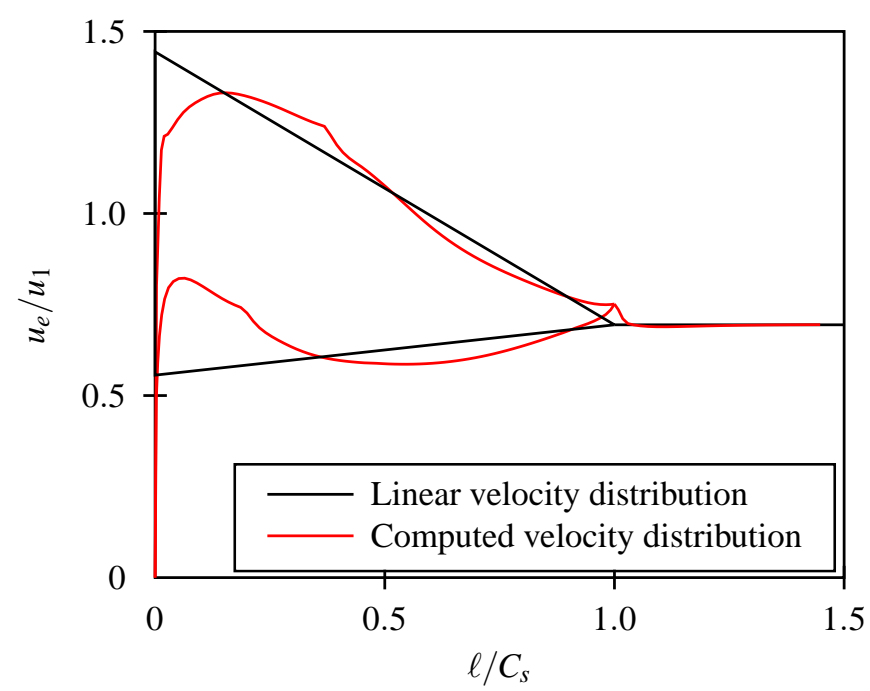

FIGURE 3: MODEL ASSUMED VELOCITY DISTRIBUTION COMPARED TO CALCULATED CASCADE VELOCITY DISTRIBUTION

satisfying conservation of angular momentum through the blade row given the blade spacing.

Figure 3 shows a comparison of this velocity distribution to a velocity distribution computed using a $2 \mathrm{D}$ viscous-inviscid cascade flow solver [15] for a representative compressor airfoil cascade. Although the linear velocity distribution is a simple estimate, it captures the peak overspeed and the slope of the suction side deceleration, which have the largest effect on profile losses.

The end wall velocity is assumed to increase linearly from suction side to pressure side, with the rotor casing velocity taken as the vector sum of the velocity in the blade-relative frame and the rotor tip speed.

\section{LOSS MECHANISMS}

As in Equation (1), the efficiency is directly related to the irreversible increase of entropy. For incompressible, adiabatic flow, the entropy rise is related to the mechanical dissipation, $\Phi$, as

$$
\dot{m} T_{t 2} \Delta s=\Phi=\iiint(\overline{\bar{\tau}} \cdot \nabla) \cdot \mathbf{u} d V
$$

In Equation (3), the term $\overline{\bar{\tau}}$ is the stress tensor, and $\mathbf{u}$ is the velocity vector. The dissipation is the rate of change of mechanical energy to heat due to the action of viscous stresses. In the analysis, it is not necessary to perform the volume integral of Equation (3) because, if a control volume is appropriately chosen, the dissipation can be determined in terms of the mechanical energy flux at the control volume boundaries [16].

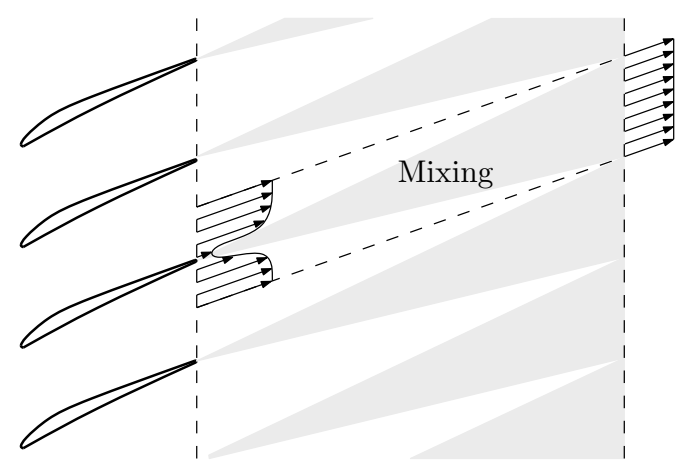

FIGURE 4: WAKE MIXING DOWNSTREAM OF A BLADE ROW

\section{Boundary layer dissipation}

The viscous dissipation is estimated from two-dimensional turbulent boundary layer theory as the product of local dissipation per unit area and blade wetted area. This approach has been used by Denton to good effect (see reference [5] for additional comments).

The dissipation per unit depth, $\Phi^{\prime}$, for a two-dimensional boundary layer can be expressed in terms of integral boundary layer properties at a streamwise location, $\ell$ [16].

$$
\Phi^{\prime}(\ell)=\int_{0}^{\ell} \rho_{e} u_{e}^{3} C_{D} d \ell=\frac{1}{2} \rho_{e}(\ell) u_{e}(\ell)^{3} \theta^{*}(\ell)
$$

In Equation (4), $C_{D}$ is the dissipation coefficient, and $\theta^{*}$ is the boundary layer kinetic energy thickness. If the surface velocity distribution $u_{e}(\ell)$ is known, an integral boundary layer method provides a rapid and useful way to determine the evolution of boundary layer quantities and thus loss. For this, closure correlations as described in [17] are needed to relate $C_{D}$ to the momentum thickness $\theta$ and boundary layer shape factor $H$. The use of the dissipation coefficient is advantageous because its variation with shape factor is much less than the skin friction coefficient. Over the range $300<\operatorname{Re}_{\theta}<1000$ and $1<H<2$, the dissipation coefficient can be approximated as constant, and the loss per unit surface area is proportional to the cube of the velocity [5] [17].

$$
\Phi \approx 0.002 \iint \rho_{e} u_{e}^{3} d A
$$

Equation (5) is used for estimates of dissipation in the hub and casing boundary layers.

\section{Wake mixing dissipation}

The downstream wake mixing losses can be evaluated using the control volume shown in Figure 4, which is bounded by periodic streamlines and extends from the blade row trailing edge 


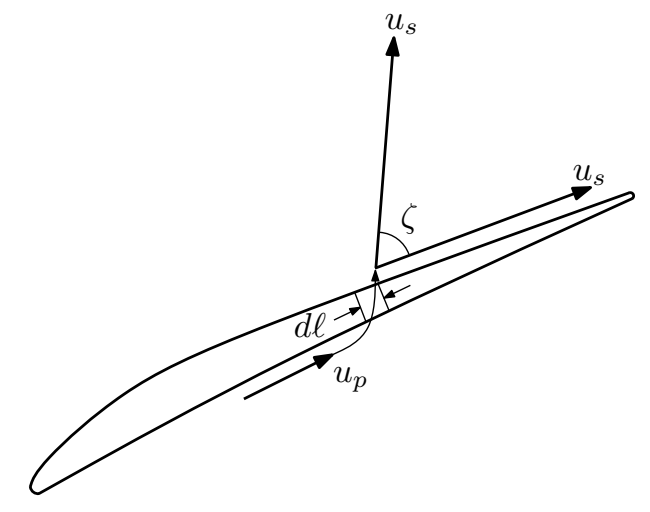

FIGURE 5: TIP LEAKAGE FLOW OVER SMALL CHORD LENGTH $d \ell$

to a downstream location where the flow properties are uniform. Given the conditions at the trailing edge, the mixing dissipation can be found by applying conservation of mass and momentum.

\section{Leakage flow dissipation}

The third loss source considered is that due to mixing of tip leakage flow with the main flow on the suction side of the rotor blade tip. Consistent with the ideas expressed above, we use a control volume analysis [5] based on the following assumptions:

1. the flow through the clearance gap prior to mixing is isentropic and driven by the static pressure difference across the blade, and

2. the clearance flow is small relative to the main flow and mixing loss occurs at the gap exit conditions. ${ }^{2}$

Figure 5 shows the leakage flow over a portion of the blade tip $d \ell$. The loss is modeled as mixing of an incremental mass injection, $d \dot{m}$, with the main flow, both having velocity $u_{s}$ and a mixing angle $\zeta$. The mixing loss is equal to the kinetic energy lost by the leakage flow as it equilibrates with the suction side flow.

$$
d \Phi=\frac{1}{2} u_{s}^{2}\left[\sin ^{2} \zeta+(1-\cos \zeta)^{2}\right] d \dot{m}
$$

The total leakage loss is obtained by integrating Equation (6) over the chord length, with the local distributions of velocity, $u(\ell)$, mixing angle, $\zeta(\ell)$, and leakage mass flow, $d \dot{m}(\ell)$, determined by the blade pressure and suction side velocity distributions.

\footnotetext{
${ }^{2}$ This assumption appears to hold well for compressors, but not for turbine leakage flows in which the vortex dynamics play a larger role [18]
}

TABLE 1: STAGE EFFICIENCY CALCULATION INPUTS

\begin{tabular}{lcl}
\hline \hline Flow coefficient & $\phi=u_{x} / \omega r$ & independent \\
Stage loading coefficient & $\psi=\Delta h_{t} /(\omega r)^{2}$ & independent \\
Reynolds number & $\mathrm{Re}=u_{x} c / v$ & design parameter \\
Aspect ratio & $\mathrm{AR}=\left(r_{o}-r_{i}\right) / c$ & design parameter \\
Hub-to-tip ratio & $r_{i} / r_{o}$ & design parameter \\
Gap-to-height ratio & $\tau /\left(r_{o}-r_{i}\right)$ & design parameter \\
Inter-stage swirl & $\alpha_{1}$ & design variable \\
Solidity & $\sigma=c / s$ & design variable \\
\hline \hline
\end{tabular}

\section{STAGE EFFICIENCY}

\section{Parametric dependence and optimization}

Combining Equations (1) and (3), the loss in stage efficiency is expressed as the sum of the dissipation due to the different loss sources divided by the stage work.

$$
\eta=1-\frac{\sum \Phi}{\dot{m} \Delta h_{t}}
$$

The dissipation terms can be found in terms of the input parameters listed in Table 1. The details of the calculation are given in Appendix A, but we can state the parametric dependence as

$$
\eta=F\left(\phi, \psi, \operatorname{Re}, \mathrm{AR}, r_{i} / r_{o}, \tau /\left(r_{o}-r_{i}\right), \alpha_{1}, \sigma\right)
$$

Calculations of efficiency over a range of inputs show $\alpha_{1}$ and $\sigma$ take on values that maximize efficiency given values for the other inputs. These parameters are thus considered as design variables, which can be solved for, rather than specified, by optimization for maximum $\eta$. The parameters $\operatorname{Re}, \mathrm{AR}, r_{i} / r_{o}$, and $\tau /\left(r_{o}-r_{i}\right)$ will, in general, be fixed by constraints additional to aerodynamic performance. They can therefore be considered as the design parameters, which are fixed inputs for a given design (i.e., for a certain stage of a machine operating at a specific set of conditions). The remaining inputs, $\phi$ and $\psi$, are independent variables. Equation (8) can then be rewritten as

$$
\left[\eta_{\max }, \alpha_{1}^{*}, \sigma^{*}\right]=F^{*}\left(\phi, \psi ;\left[\operatorname{Re}, \mathrm{AR}, r_{i} / r_{o}, \tau /\left(r_{o}-r_{i}\right)\right]\right),
$$

with results expressed as contours of maximum efficiency and optimal design variables as a function of $\phi$ and $\psi$ for a given combination of the bracketed terms.

While conceptually, the optimization implied in Equation (9) is straightforward, practical implementation is more challenging. For moderate to high stage loadings, at least one blade row 


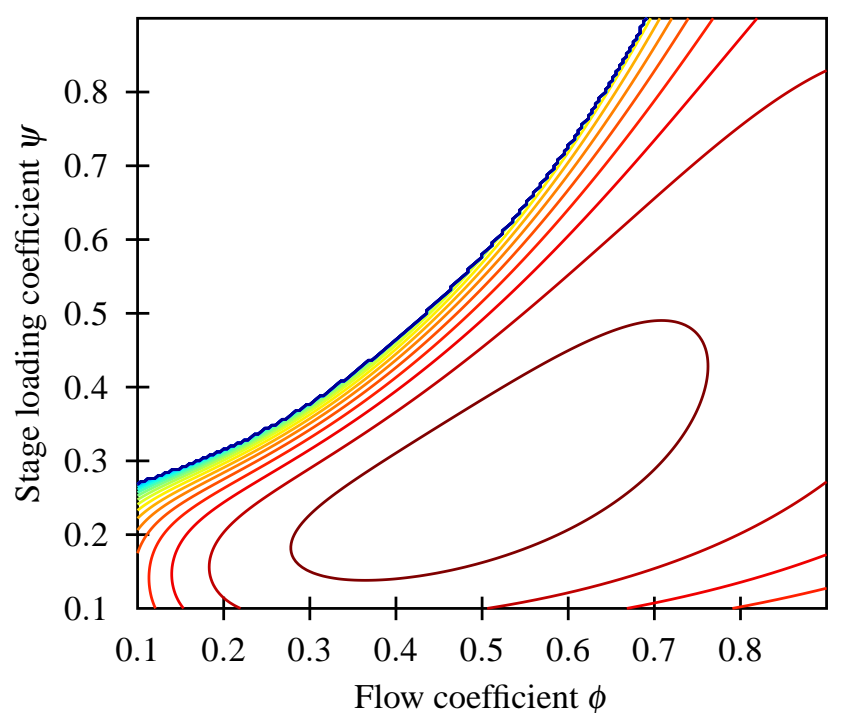

FIGURE 6: EFFICIENCY OF REPRESENTATIVE COMPRESSOR STAGE; CONTOUR INCREMENT: $1 \%$, PEAK CONTOUR: $95 \%$

of the optimal stage is operating near or at the separation limit (i.e., increases in the turning or blade spacing result in separation). The functional dependence of efficiency on the design variables at this limit is such that multiple local maxima in efficiency occur. However, because the efficiency is not sensitive to changes in blade spacing and stage reaction near the separation limit, we can cast the optimization in terms of stages with specified diffusion factor, $D$, and stage reaction, $\Lambda$. More specifically, for the blade velocity profile distribution used, a reaction of 0.5 minimizes the overspeeds and suction side deceleration and corresponding profile losses in both blade rows. Detailed computations (which are the principle justifcation for the approximation made) show that stages with diffusion factor of 0.45 and with reaction of 0.5 provide an excellent estimate for the optimum, since the change in efficiency from these values to the optimized case is on the order of $0.1 \%$ [19].

\section{A representative stage}

To show the procedure in a more concrete fashion, we present results for stage efficiency for a representative aero engine high pressure compressor with the following design parameters.

$\begin{array}{lc}\mathrm{Re} & 500,000 \\ \mathrm{AR} & 2.25 \\ r_{i} / r_{o} & 0.65 \\ \tau /\left(r_{o}-r_{i}\right) & 0.01 \\ D & 0.45 \\ \Lambda & 0.5\end{array}$

TABLE 2: MAGNITUDES OF LOSSES AT PEAK EFFICIENCY

\begin{tabular}{ll}
\hline \hline Loss source & $\Delta \eta(\%)$ \\
\hline Rotor blade boundary layer & 1.00 \\
Rotor wake mixing & 0.23 \\
Rotor end wall boundary layer & 0.96 \\
Rotor tip clearance & 0.41 \\
Stator vane boundary layer & 1.00 \\
Stator wake mixing & 0.23 \\
Stator end walls & 0.73 \\
\hline Total & 4.55 \\
\hline \hline
\end{tabular}

Figure 6 shows contours of efficiency versus flow coefficient and stage loading coefficient, and we make three observations about the results. First, the peak efficiency is $95.4 \%$. This can be compared to stage efficiencies of $92 \%$ quoted in the open literature [20], although we recognize that much of the relevant information on this point resides within industry. Second, there exists a region of high loading and low flow coefficient (the blank region in the upper left-hand corner of Figure 6) where attached flow cannot be maintained over the entire blade profile. Even with the solidity set by a fixed diffusion factor, beyond a certain loading, the suction side deceleration is too large, and the flow separates. Third, the peak efficiency occurs with flow coefficient in the range of 0.4-0.6 and stage loading coefficient between 0.2 and 0.3. In this range, values of de Haller number $\left(u_{\text {out }} / u_{\text {in }}\right)$ are near 0.75 , in agreement with observations from cascade theory. At higher loadings, losses increase more rapidly than the stage work. At loadings below the range of peak efficiency, losses remain nearly constant as the work decreases, resulting in lower efficiencies. Similar trends with stage loading and flow coefficients are seen in existing data for compressor stage efficiency, although, in line with the aim of the paper, the levels in Figure 6 are higher by several points than quoted results [4]. The trends also agree with findings of Dickens and Day [14], who concluded that increasing stage loading to values above that of conventional designs ( 0.2 to 0.4$)$ led to decreased stage efficiency.

Table 2 shows the breakdown of loss sources in the rotor and stator. The even split between rotor and stator profile losses is due to the $50 \%$ stage reaction. The end wall boundary layer losses in the rotor are larger than in the stator because the velocity on the rotor casing (the vector sum of the blade-relative velocity distribution and the rotor tip speed) is higher than stator casing velocity (which for the 50\% reaction case is the same as the rotor blade-relative velocity distribution). At the design 
point considered, the end wall area is approximately equal to the blade and vane surface area, and the boundary layer losses of each are roughly equal as well; this would suggest that, under the assumptions of the model, for well-designed stages, all skin friction losses are proportional to the wetted surface area. The downstream wake mixing loss is approximately $20 \%$ of the total profile loss for rotor and stator. The tip clearance loss is about $10 \%$ of the total stage loss, corresponding to a loss of a half percent in stage efficiency for one percent non-dimensional blade height.

\section{Impact of design parameters}

The sensitivity of stage efficiency to design parameters can be evaluated by considering the dependence of each in the components of the efficiency formulation (given in Appendix A). Numerical determination of these sensitivities is complicated (e.g., the dependence on Reynolds number, since the profile losses are determined using an integral boundary layer method with Reynolds-dependence closure relations) and varies with the choice of inputs. Useful information can be obtained, however, from considering the approximate scalings of efficiency with respect to each individual design parameter. For example, because the profile boundary layers are taken as turbulent, the boundary layer thicknesses and corresponding boundary layer and wake losses are proportional to $\mathrm{Re}^{-1 / 5}$, a scaling inherent in the turbulent closure relations of the integral boundary layer method.

Aspect ratio and hub-to-tip radius ratio, the parameters that characterize the annular geometry, determine the end wall surface area per unit mass flow. End wall loss is inversely proportional to aspect ratio, since, if all other variables are held constant, an increase in aspect ratio shrinks the axial extent and thus the relative end wall area of the blade rows. High aspect ratios have been observed, however, to be susceptible to other performance issues [21], we thus confine our examination to the parameter space of modern compressor aspect ratios. The hub-totip radius ratio has a small effect on the relative end wall area, since the changes in relative hub and casing area roughly offset. Hub-to-tip ratio can, however, have a strong effect on rotor casing loss, since changes in $r_{i} / r_{o}$ can increase or decrease the vector sum of the blade-relative velocity and the tip speed of the rotor.

The tip clearance loss model neglects interactions of the end wall and clearance flows, and thus does not capture the increase in losses at very small clearances (below roughly $0.5 \%$ of height) and the resulting optimal gap height [9]; outside this range of clearances, however, the linear trend with gap height is in accord with experience, though at a smaller rate [22] (see Appendix A for a fuller description of the leakage mixing model).

Table 3 shows the peak efficiency for a few representative stages. The design parameters are meant to represent specific stages of a moderate bypass ratio engine for a single-aisle com-
TABLE 3: CALCULATED MAXIMUM EFFICIENCY OF STAGES REPRESENTING DIFFERENT AERO ENGINE COMPRESSOR STAGE TYPES

\begin{tabular}{llllll}
\hline \hline & $\mathrm{Re}$ & $\mathrm{AR}$ & $r_{i} / r_{o}$ & $\tau /\left(r_{o}-r_{i}\right)$ & $\eta(\%)$ \\
\hline First LPC & $1.0 \times 10^{6}$ & 2.5 & 0.8 & 0.01 & 96.1 \\
First HPC & $0.5 \times 10^{6}$ & 2.25 & 0.65 & 0.01 & 95.4 \\
Last HPC & $1.0 \times 10^{6}$ & 1.25 & 0.9 & 0.05 & 93.1 \\
\hline \hline
\end{tabular}

mercial airliner. It is worth stating the assumptions and losses included in the model will have varying levels of applicability depending on the stage. For instance, a compressor first stage can have incoming relative Mach numbers that lead to shocks and increased profile losses, and will likely have a higher reaction than $50 \%$. The result here is thus an optimistic estimate of the efficiency in such a stage. When compared to other similar stages, however, the trends can give useful insight into the impact of factors that are included in the model (e.g., choice of annular geometry).

Some of the trends described above are apparent, such as the decrease in efficiency with tip clearance height (here, the non-dimensional clearance height has been estimated assuming a fixed dimensional clearance height, leading to the large clearance in the final stage of the high pressure compressor). The behavior of efficiency with loading and flow coefficients for these designs have been seen to be similar to that seen in Figure 6. For design parameters spanning the ranges in modern aero engine compressors, the peak efficiency is not particularly sensitive to Reynolds number or the annular stage geometry, which account for changes in efficiency of less than $1 \%$ over the range of designs presented in Table 3.

\section{IMPLICATIONS FOR CYCLE PERFORMANCE}

To provide context for the stage results, we present the sensitivity of engine performance to compressor efficiency. We consider the thermal efficiency of an uncooled ${ }^{3}$ Brayton cycle, which can serve as a surrogate for the gas generator of an aero engine ${ }^{4}$ or other gas turbine application.

\footnotetext{
${ }^{3}$ From a theoretical cycle performance standpoint, it has been shown that the presence of cooling flow has no effect on thermal efficiency [23]. In practice, cooling flows may introduce additional losses in the engine [8], and an uncooled cycle can be thought of as representing a cooled cycle with a given level of cooling and material technology.

${ }^{4}$ In this context, the gas generator consists of the inner annulus of the fan through which the core flow flows, the compressors, combustor, and the portion of the turbine providing work for the compressors and inner annulus of the fan. This definition allows a separation of the gas generator thermal efficiency (which depends on component efficiencies) from the propulsive efficiency (which is driven by the fan pressure ratio).
} 


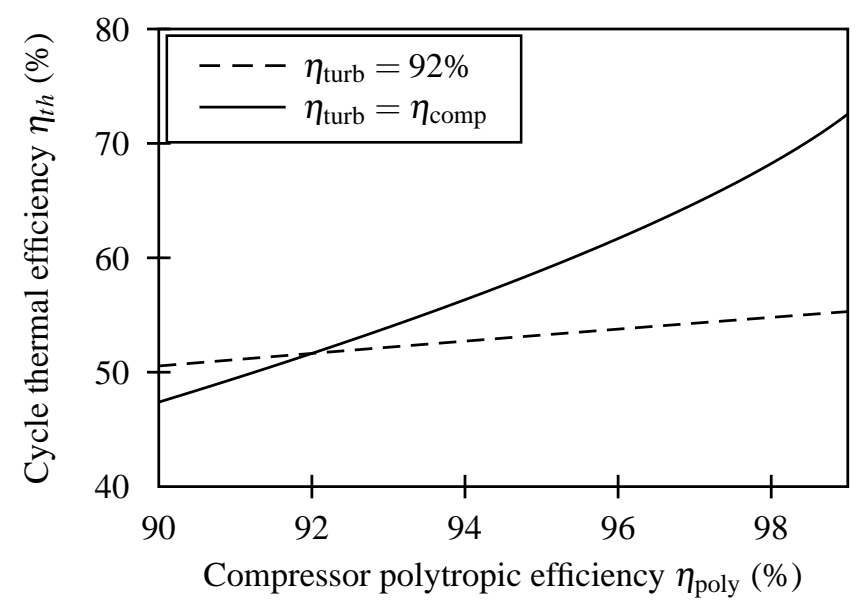

FIGURE 7: GAS GENERATOR THERMAL EFFICIENCY VERSUS COMPRESSOR EFFICIENCY

For a given cycle temperature ratio, $\theta_{t}$, and component efficiencies, there exists an optimal pressure ratio that maximizes thermal efficiency. The cycle thermal efficiency is thus presented as a function of component efficiency with $\theta_{t}=5.5$, representing a contemporary commercial aero engine at cruise [24]. Figure 7 shows cycle efficiency versus compressor polytropic efficiency for a fixed turbine efficiency of $92 \%$. Turbine stage analysis similar to the analysis presented in this paper suggests that similar advances in turbine efficiency may also be possible [19], so cycle efficiency for equal compressor and turbine efficiencies is also shown. The cycle efficiency is calculated assuming the flow through the engine is a perfect gas with constant specific heat, starting at atmospheric conditions. At each point, the cycle pressure ratio has been optimized for maximum thermal efficiency. Both curves exhibit a more than linear increase in thermal efficiency with polytropic efficiency. For constant turbine efficiency, a one percent increase in compressor efficiency results in an increase in cycle thermal efficiency of about $0.5 \%$. If turbine efficiency is assumed to increase comparable to compressor efficiency, a one percent increase in component efficiency results in an increase in cycle thermal efficiency of about $2.5 \%$.

Given the stage performance results listed in Table 3, and accounting for the assumptions of the model, it seems reasonable to assume that an advanced compressor might have a theoretical maximum polytropic efficiency as high as $95 \%$. Assuming a baseline polytropic efficiency of $92 \%$ in both the compressor and turbine, this increase in compressor efficiency alone would result in an increase of $1.6 \%$ in cycle efficiency, and increasing both compressor and turbine efficiency to $95 \%$ would allow an increase of thermal efficiency of up to $7.0 \%$ for fixed turbine inlet temperature, with potential for larger increases as the turbine temperature is increased. Increased component efficiency is only one enabling technology required for these advanced cycles; the pressure ratios for the advanced cycles are as high as 100 , well beyond those of conventional machines. Two major challenges arise in these types of machines: the first is increased compressor exit temperature, the second is maintaining a high level of compressor efficiency as the compressor exit corrected flow and compressor blade heights decrease at the back of the machine.

\section{SUMMARY AND CONCLUSIONS}

A framework has been presented for estimating the limits of axial compressor aerodynamic performance. In the analysis, a rotor-stator geometry and an assumed surface velocity distribution are used to represent a generic compressor stage. Sources of irreversibility are assessed using basic control volume and boundary layer analyses, rather than by extrapolating data from existing machines.

The aim of the study was to determine an upper bound on compressor stage efficiency, and this has been accomplished through selection of the loss mechanisms that are included. By considering different loss sources under different assumptions, however, the analytical framework can be adjusted to determine possible avenues for performance improvement in different types of stages.

Table 3 gives estimates of the upper bound on efficiency for a range of stage designs. Although achieving these levels in practice is indeed a stretch goal, the results show what advances are possible. The trend in efficiency with stage loading and flow coefficients in Figure 6 is similar over the design parameters considered, with peak efficiency occurring at stage loading coefficients between 0.2 and 0.4 , where the stage profiles have properties in ranges known to be efficient. This observation, coupled with the similarities in the shape of the "Smith chart" to that developed using empirical information [4] seems to indicate that the losses included are dominant in determining the efficiency trends with respect to basic stage design parameters.

Figure 7 shows that component performance increases that approach the estimated limits of component efficiency result in substantial increases in overall engine thermal efficiency $(51.6 \%$ to $58.9 \%$ for the case examined). Technological challenges arising from such advanced cycles include increased compressor exit temperature and the maintainance of compressor efficiency as the exit corrected flow decreases with size and increased pressure ratio.

\section{ACKNOWLEDGMENT}

This research was sponsored by the Fundamental Aeronautics Program at the NASA Glenn Research Center under Cooperative Agreement Number NNX08AW63A. The authors would also like to acknowledge J. J. Adamczyk, N. A. Cumpsty, M. Drela, and W. K. Lord, for their contributions throughout the duration of this study, and A. H. Epstein, N. A. Fitzgerald, A. 
Huang, G. F. Kiwada, J. S. Sabnis, and J. B. Staubach for helpful comments and suggestions.

\section{REFERENCES}

[1] Koch, C. C., and Smith, L. H., 1967. "Loss Sources and Magnitudes in Axial-Flow Compressors". Journal of Engineering for Power, 98(3), July, pp. 411-424.

[2] Koch, C. C., 1981. "Stalling Pressure Rise Capability of Axial Flow Compressor Stages". Journal of Engineering for Power, 103, pp. 645-656.

[3] Leroy H. Smith, J., 2002. "Axial Compressor Aerodesign Evolution at General Electric". Journal of Turbomachinery, 124, July, pp. 321-330.

[4] Wright, P. I., and Miller, D. C., 1991. "An Improved Compressor Performance Prediction Model". In European Conference of Turbomachinery: Latest Developments in a Changing Scene, IMechE.

[5] Denton, J. D., 1993. "Loss Mechanisms in Turbomachines". Journal of Turbomachinery, 115, October, pp. 621-656.

[6] Storer, J. A., and Cumpsty, N. A., 1994. “An Approximate Analysis and Prediction Method for Tip Clearance Loss in Axial Compressors". Journal of Turbomachinery, 116, October, pp. 648-656.

[7] Yaras, M. I., and Sjolander, S. A., 1992. "Prediction of Tip-Leakage Losses in Axial Turbines". Journal of Turbomachinery, 114, January, pp. 204-210.

[8] Young, J. B., and Wilcock, R. C., 2002. "Modeling the AirCooled Gas Turbine: Part 2-Coolant Flows and Losses". Journal of Turbomachinery, 124, April, pp. 214-221.

[9] Cumpsty, N. A., 1989. Compressor Aerodynamics. Longman Group UK Ltd.

[10] Lieblein, S., and Stockman, N. O., 1972. "Compressibility Correction for Internal Flow Solutions". Journal of Aircraft, 9(4), April, pp. 312-313.

[11] Halstead, D. H., Wisler, D. C., Okiishi, T. H., Walker, H. P., Hodson, H. P., and Shin, H.-W., 1997. "Boundary Layer Development in Axial Compressors and Turbines: Part 1 of 4 - Composite Picture". Journal of Turbomachinery, 119, January, pp. 114-127.

[12] Hodson, H. P., and Howell, R. J., 1992. "Bladerow Interactions, Transition, and High-Lift Aerofoils in Low-Pressure Turbines". Journal of Engineering for Gas Turbines and Power, 114, October, pp. 603-611.

[13] Smith, L. H., 1966. "Wake Dispersion in Turbomachines". Journal of Basic Engineering, 88, pp. 688-690.

[14] Dickens, T., and Day, I., 2011. "The Design of Highly Loaded Axial Compressors". Journal of Turbomachinery, 133, July.

[15] Drela, M., 1986. Two-Dimensional Transonic Aerody- namic Design and Analysis Using the Euler Equations. GTL Report 187, Massachusetts Institute of Technology.

[16] Drela, M., 2009. "Power Balance in Aerodynamics Flows". AIAA Journal, 47(7), July, pp. 1761-1771.

[17] Drela, M., and Giles, M. B., 1987. "Viscous-Inviscid Analysis of Transonic and Low Reynolds Number Airfoils". AIAA Journal, 25(10), October, pp. 1347-1355.

[18] Huang, A., 2011. "Loss Mechanisms in Turbine Tip Clearance Flow". MS Thesis, Massachusetts Institute of Technology, Cambridge, MA, May.

[19] Hall, D. K., 2011. "Performance Limits of Axial Turbomachine Stages". MS Thesis, Massachusetts Institute of Technology, Cambridge, MA, February.

[20] Benzakein, M. J., 2001. "Propulsion Strategy for the $21^{\text {st }}$ Century: A Vision Into the Future".

[21] Wennerstrom, A. J., 1989. "Low Aspect Ratio Axial Flow Compressors: Why and What It Means". Journal of Turbomachinery, 111, October, pp. 357-365.

[22] Wisler, D. C. Advanced compressor and fan systems. Lecture notes.

[23] Horlock, J. H., 2003. Advanced Gas Turbine Cycles. Pergamon.

[24] Cumpsty, N. A., 2003. Jet Propulsion, 2nd ed. Cambridge University Press.

\section{Appendix A: Stage Efficiency Calculation}

The loss in stage efficiency is calculated as the sum of the efficiency debits due to the four loss sources considered.

$$
\eta=1-\left(\Delta \eta_{\text {surf }}+\Delta \eta_{\text {wake }}+\Delta \eta_{\text {wall }}+\Delta \eta_{\text {tip }}\right)
$$

This amounts to seven terms that need to be calculated: the $\Delta \eta$ 's in both the rotor and the stator for blade boundary layer, end wall boundary layer, and wake mixing dissipation, and the rotor clearance flow mixing loss.

\section{Surface velocity distribution.}

Each of the loss sources depends on the assumed surface velocity distribution. The velocity triangles of the stage profile (see Figure 2a) can be determined in terms of $\phi, \psi$, and either a specified incoming absolute swirl angle, $\alpha_{1}$ or stage reaction, $\Lambda$.

$$
\begin{aligned}
& \alpha_{1}=\left\{\begin{array}{l}
\alpha_{1, \mathrm{spec}} \\
\tan ^{-1}\left(\frac{2\left(1-\Lambda_{\mathrm{spec}}\right)-\psi}{2 \phi}\right)
\end{array}\right. \\
& \alpha_{2}=\tan ^{-1}\left(\frac{\psi}{\phi}+\tan \alpha_{1}\right) \\
& \alpha_{3}=\alpha_{1}
\end{aligned}
$$




$$
\begin{aligned}
& \beta_{1}=\tan ^{-1}\left(\frac{1}{\phi}-\tan \alpha_{1}\right) \\
& \beta_{2}=\tan ^{-1}\left(\frac{1}{\phi}-\tan \alpha_{2}\right)
\end{aligned}
$$

Both the rotor blades and stator vanes are assumed to have circular arc camber lines. The camber length (which, under the assumption of thin blades, is a good approximation to streamwise surface length), is then given by

$$
\left(\frac{C_{s}}{c}\right)=\frac{\frac{1}{2}\left(\alpha_{\text {in }}-\alpha_{\text {out }}\right)}{\sin \left(\frac{1}{2}\left(\alpha_{\text {in }}-\alpha_{\text {out }}\right)\right)},
$$

where $\alpha_{\text {in }}$ is the blade-relative incoming flow angle ( $\beta_{1}$ for the rotor and $\alpha_{2}$ for the stator) and $\alpha_{\text {out }}$ is the blade-relative exit flow angle ( $\beta_{2}$ for the rotor and $\alpha_{3}$ for the stator).

The blade spacing is set by specification of either the solidity $\sigma$ of a blade row or a diffusion factor $D$ (from which $\sigma$ is calculated explicitly).

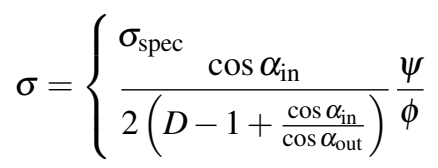

The linear profile velocity distribution can be determined from the velocity triangles, blade shape, and blade spacing.

$$
\frac{u(\ell)}{u_{\mathrm{in}}}=\left(1-\frac{\ell}{C_{s}}\right)\left(1 \pm \frac{\cos \alpha_{\mathrm{in}}}{2 \sigma\left(C_{s} / c\right)} \frac{\psi}{\phi}\right)+\frac{\ell}{C_{s}} \frac{\cos \alpha_{\mathrm{in}}}{\cos \alpha_{\mathrm{out}}}
$$

The blade-relative end wall velocity distribution is assumed to vary linearly across the blade pitch from suction to pressure side.

$$
u_{\mathrm{rel}}=u_{p}\left(x^{\prime}\right)+y^{\prime}\left(u_{s}\left(x^{\prime}\right)-u_{p}\left(x^{\prime}\right)\right)
$$

where $u_{s}$ and $u_{p}$ are the profile suction and pressure side velocities defined in Equation (18), and $x^{\prime}=x / c_{x}$ is the non-dimensional axial direction, and $y^{\prime}=r \theta / s(r)$ is the nondimensional pitch-wise direction on the wall being considered. For the rotor hub and both stator end walls, $u_{\mathrm{wall}}=u_{\mathrm{rel}}$. The velocity on the rotor casing is estimated by vector addition of the blade tip velocity and the blade-relative end wall velocity $u_{\text {rel }}$, assuming the direction of $u_{\text {rel }}$ is equal to the blade stagger angle $\xi$.

$$
u_{\mathrm{wall}}=u_{\mathrm{rel}} \cos \xi \sqrt{1+\left(\frac{1}{\phi\left(u_{\mathrm{rel}} / u_{x}\right) \cos \xi}-\tan \xi\right)^{2}}
$$

\section{Blade boundary layer dissipation.}

The losses associated with blades and vanes is approximated using the two-dimensional stage profile. An integral boundary layer method is used to solve numerically for the trailing edge boundary layer quantities in terms of the blade velocity distribution and Reynolds number. ${ }^{5}$

$$
F\left(\frac{u(\ell)}{u_{\text {in }}}, \operatorname{Re}\right) \longrightarrow\left[\frac{\delta_{\mathrm{TE}}^{*}}{C_{s}}, \frac{\theta_{\mathrm{TE}}}{C_{s}}, \frac{\theta_{\mathrm{TE}}^{*}}{C_{s}}\right]
$$

Applying Equation (4), the associated loss is determined in terms of the calculated boundary layer kinetic energy thickness and the profile geometry.

$$
\Delta \eta_{\mathrm{surf}}=\frac{1}{2} \sigma\left(\frac{C_{s}}{c}\right)\left(\frac{1}{\cos \alpha_{\mathrm{out}}}\right)^{3} \frac{\theta_{\mathrm{TE}}^{*}}{C_{s}} \frac{\phi^{2}}{\psi} .
$$

\section{End wall boundary layer dissipation.}

The boundary layer loss is estimated assuming a constant dissipation coefficient with the end wall velocity distribution, $u_{\text {wall }}\left(x^{\prime}, y^{\prime}\right)$.

$$
\Delta \eta_{\text {wall }}=C_{D} \frac{\phi^{2}}{\psi} \frac{\cos \xi}{\mathrm{AR}} \frac{2}{1+\left(r_{i} / r_{o}\right)^{ \pm 1}} \int_{0}^{1} \int_{0}^{1}\left(\frac{u_{\mathrm{wall}}\left(x^{\prime}, y^{\prime}\right)}{u_{x}}\right)^{3} d x^{\prime} d y^{\prime},
$$

In the present analysis, the integral in Equation (23) is estimated using $5 \times 5$ point Gaussian quadrature.

\section{Wake mixing dissipation.}

The wake mixing loss is determined using a control volume analysis of the downstream mixing region shown in Figure 4. Conservation of mass and momentum are used to determine the change of flow angle and the static pressure drop due to mixing in terms of boundary layer quantities. Once the velocities and pressures downstream are known, the loss can be determined in terms of the trailing edge boundary layer quantities by applying Equation (3).

$$
\begin{aligned}
\Delta \eta_{\text {wake }} & =\frac{\phi^{2}}{\psi}\left\{\left[M-(1-D)+(1-D)^{2}\right](1-D)\right. \\
& \left.+\frac{1}{2}\left[\frac{(1-D)-K}{\cos ^{2} \alpha_{\text {out }}}-\frac{(1-D)^{3}}{\cos ^{2} \alpha_{m}}\right]\right\}
\end{aligned}
$$

\footnotetext{
${ }^{5} \mathrm{We}$ do not present the details of the numerical computations or the boundary layer method here. For details of a similar implementation, see reference [17].
} 
where $D, M$, and $K$ are related to the boundary layer displacement, momentum, and kinetic energy thicknesses,

$$
\begin{aligned}
& D=\left(\frac{\delta_{\mathrm{TE}}^{*}}{C_{s}}\right)\left(\frac{C_{s}}{c}\right) \frac{\sigma}{\cos \alpha_{\mathrm{out}}}, \\
& M=\left(\frac{\theta_{\mathrm{TE}}}{C_{s}}\right)\left(\frac{C_{s}}{c}\right) \frac{\sigma}{\cos \alpha_{\mathrm{out}}}, \\
& K=\left(\frac{\theta_{\mathrm{TE}}^{*}}{C_{s}}\right)\left(\frac{C_{s}}{c}\right) \frac{\sigma}{\cos \alpha_{\mathrm{out}}},
\end{aligned}
$$

and the mixed-out flow angle is given by

$$
\alpha_{m}=\tan ^{-1}\left[\tan \alpha_{\text {out }} \frac{1-D-M}{(1-D)^{2}}\right]
$$

\section{Tip clearance flow dissipation.}

With the assumption of isentropic flow through the rotor tip clearance, Equation (6) can be rewritten as

$$
d \Phi=u_{s}\left(u_{s}-u_{p}\right) d \dot{m} .
$$

The leakage mass flow, driven by the pressure difference, is given by

$$
d \dot{m}=\rho C_{d} \tau \sqrt{u_{s}^{2}-u_{p}^{2}} d z
$$

where $C_{d}$ is a discharge coefficient to account for the detailed behavior of the flow within the gap (a value of 0.8 is seen to give results that match experimental data [6]).

Given an assumed tip velocity distribution, Equation (29) can be integrated along the blade chord and divided by the stage work to determine the loss in efficiency. Rather than use the assumed profile distribution, a rectangular distribution was used.

$$
\begin{aligned}
& u_{s}-u_{p} \approx u_{x} \frac{\tan \alpha_{2}-\tan \alpha_{1}}{\sigma} \\
& u_{s}+u_{p} \approx u_{x} \frac{2}{\cos \xi}
\end{aligned}
$$

This gives the same blade loading at the tip while minimizing the clearance loss. The resulting loss in efficiency can then be expressed as

$$
\Delta \eta_{\text {tip }}=C_{d}\left(\frac{\tau}{r_{o}-r_{i}}\right)\left(\frac{C_{s}}{c}\right) \frac{2 \phi \sqrt{\psi}(\psi \cos \xi+2 \phi \sigma)}{(2 \phi \sigma \cos \xi)^{3 / 2}}
$$

